\title{
Electrochemical polymerization of conducting polymers in living neural tissue
}

\author{
Sarah M Richardson-Burns ${ }^{1,2}$, Jeffrey L Hendricks ${ }^{3}$ and \\ David C Martin ${ }^{2,3,4}$ \\ ${ }^{1}$ Regenerative Sciences Program, University of Michigan, Ann Arbor, MI 48109, USA \\ ${ }^{2}$ Departments of Materials Science and Engineering, University of Michigan, Ann Arbor, MI 48109, \\ USA \\ ${ }^{3}$ Biomedical Engineering, University of Michigan, Ann Arbor, MI 48109, USA \\ ${ }^{4}$ Macromolecular Science and Engineering Center, University of Michigan, Ann Arbor, MI 48109, USA
}

Received 7 August 2006

Accepted for publication 14 December 2006

Published 2 February 2007

Online at stacks.iop.org/JNE/4/L6

\begin{abstract}
A number of biomedical devices require extended electrical communication with surrounding tissue. Significant improvements in device performance would be achieved if it were possible to maintain communication with target cells despite the reactive, insulating scar tissue that forms at the device-tissue interface. Here, we report that the conducting polymer poly(3,4-ethylenedioxythiophene) (PEDOT) can be polymerized directly within living neural tissue resulting in an electrically conductive network that is integrated within the tissue. Nano and microscale PEDOT filaments extend out from electrode sites, presumably forming within extracellular spaces. The cloud of PEDOT filaments penetrates out into the tissue far enough that it should be possible to bypass fibrous scar tissue and contact surrounding healthy neurons. These electrically functional, diffuse conducting polymer networks grown directly within tissue signify a new paradigm for creating soft, low impedance implantable electrodes.
\end{abstract}

(Some figures in this article are in colour only in the electronic version)

\section{Introduction}

Electrically responsive tissues including the brain, heart and skeletal muscle provide a unique opportunity to couple electronic devices and computers with human or animal tissues making possible a therapeutic body-machine interface. Hence, there is growing interest in development of implantable electrode devices for diverse biomedical applications such as retinal and cochlear prosthetics, deep brain stimulation, cardiac pacemakers, drug delivery devices and biosensors. The use of electrode devices in therapies for neurologic deficits is being actively pursued with the hope of changing the prognosis for individuals with spinal cord injury, sensory or motor impairments and neurological disorders, including chronic pain, Parkinson's disease and epilepsy (Eisen and
Franck 2005, Patil et al 2004, Rodriguez-Oroz et al 2004, Stieglitz et al 2005). However, major road blocks must be overcome to make implementation of such devices possible, including limited biocompatibility and bio-integration of devices and electrodes, low-signal, non-specific interactions at the neuron-electrode interface (Polikov et al 2005), and the intellectual and technological challenge of joining electronically and ionically conductive systems (Fromherz 2002).

Neural recording and stimulating devices communicate with neurons via electrical signals and require direct, long-term functional contact with the target tissue to operate properly. Despite the privileged immunity of the central nervous system (CNS), a foreign-body response is mounted which can result in biofouling of the device, chronic inflammation, death 
of surrounding neurons and encapsulation of the device in electrically insulating glial scar tissue (Biran et al 2005, Edell et al 1992, Otto et al 2006, Rousche and Normann 1998, Szarowski et al 2003, Turner et al 1999, Williams et al 1999). Many research groups, including our own, have explored a variety of tactics for overcoming the limitations of current neural electrode devices, including attracting neurons closer to electrode sites and reducing the immuno-reactive tissue that forms around CNS implants. Schemes that have been explored include the application of rejuvenating voltage pulses (Johnson et al 2005, Vetter et al 2004), the development of devices that release anti-inflammatory drugs or neurotrophins (Jain et al 2006, Rathnasingham et al 2004, Spataro et al 2005, Williams et al 2005, Zhong and Bellamkonda 2005), modification of device electrode sites with high surface area, bioactive inherently conducting polymers (Cui et al 2003, Xiao et al 2004) and devices comprised of soft polymers (Rousche et al 2001) that buffer the tissue from inflammation-inducing micromotion (Gilletti and Muthuswamy 2006, Kim and Martin 2006, Lee et al 2005, Subbaroyan et al 2005, Yang and Martin 2006). We now propose that it may also be effective to bring the electrode out to the healthy neurons beyond the glial scar. This would reduce the need to manipulate the brain's normal healing responses or coax neurons or their processes toward the device surface. Here, we report the creation of a nano and microscale conducting polymer network that can be grown out from electrode sites of implanted devices using in vivo electropolymerization of the inherently conducting polymer, poly(3,4-ethylenedioxythiophene) (PEDOT). The PEDOT filaments integrate directly within the surrounding tissue and extend far enough out into the tissue that it should be possible to bypass the typical neuronal 'kill zone' and glial scar which have been estimated to range from 50 to $150 \mu \mathrm{m}$ (Polikov et al 2005).

\section{Results and discussion}

The generation of a conductive polymer network formed within electrically active tissue signifies a new paradigm for implantable electrodes. This research was in large part inspired by our laboratory's ongoing in vitro studies on the interactions between cultured neural cells and PEDOT toward development of a continuous electrode-neuron interface (Richardson-Burns et al 2007) as well as by previous studies showing that PEDOT and another conducting polymer, poly(pyrrole) (PPy), can be electrochemically polymerized directly within low-density hydrogels to form diffuse, filamentous conducting networks (Brahim and Guiseppi-Elie 2005, Kim et al 2000, 2004, Nyberg et al 2002). The possibility of polymerizing PPy directly within a tissue has been previously explored in porcine pericardium (Khor et al 1995). However, the resulting tissuepolymer structures did not exhibit electrical functionality and potentially cytotoxic chemical-initiated polymerization was performed.

To begin investigations into whether living neurons could be directly interfaced with PEDOT by polymerization with the conductive matrix and still maintain relatively normal physiology, we first assessed cytotoxicity of the EDOT monomer and electrochemical polymerization procedures. We found that cultured neural cells could be exposed to a $10 \mathrm{mM}$ EDOT solution (also containing $20 \mathrm{mM}$ poly(styrene sulfonate); PSS a poly-anionic dopant for PEDOT) while maintaining at least $75 \%$ viability for as long as $72 \mathrm{~h}$ (figure 1(a)) (Richardson-Burns et al 2007). Hence, the monomer-associated cytotoxicity was negligible since typical electropolymerizations had durations of 5-60 min. We also found that electrochemical polymerization procedures involving application of low electrical current $(0.5-5 \mu \mathrm{A}$ $\mathrm{mm}^{-2}$ ) were compatible for use with the neural cells since these currents did not produce unfavorable, irreversible reactions such as hydrolysis. Studies using mouse dissociated cortical neuronal cultures and SY5Y neuroblastoma-derived neural cells seeded on metal electrodes indicated that PEDOT could be electropolymerized around the cells resulting in live neural cells embedded within a three-dimensional PEDOT matrix (figure 1(b)). The morphology and topology of the PEDOT polymerized in the presence of neural cells cultured on electrodes were assessed using optical microscopy (figure 1(c)) and scanning electron microscopy (SEM; figure $1(\mathrm{~d})$ ). Prior to imaging the cells were formaldehyde fixed and for SEM the cells were also dehydrated with hexamethyldisilazane (HMDS). PEDOT formed on the electrode surface and surrounded and partially embedded the live cells and their extensions. The PEDOT was optically opaque and appeared as a dark substance with cellshaped holes which resulted from polymerization of PEDOT around the cells that were adhered to the electrode substrate (figure 1(c)) (Richardson-Burns et al 2007). SEM revealed the fuzzy, nodular nano and microscale surface morphology typical of PEDOT and indicated that the polymer also formed at the neural membrane-electrode interface and appeared to use the cells, cell membranes and cell processes as scaffolds for polymerization (figure 1(d)). The PEDOT matrix surrounding the cells was found to be $1.5-4 \mu \mathrm{m}$ thick with variations due to the presence of the cells on the electrode substrate. Interestingly, polymerization was suppressed in regions directly beneath the cells themselves, presumably because the cell membranes act as local barriers to EDOT diffusion due to strong cell-substrate adhesions (see figure 1(c)). We also found that the cell material could be removed from the PEDOT-cell matrix to result in a biomimetic conductive substrate containing cell-scale topological features such as tunnels, troughs and caves. Ongoing studies are investigating whether this kind of substrate on an implanted device encourages cells in a host tissue to re-populate the cellshaped holes and send processes into the tunnels and crevasses, making possible an intimate and specific electrical contact between the electrode and target cells.

Using methods adapted from the experiments on PEDOT polymerization in the presence of cultured neural cells, we next established the experimental conditions needed to electrochemically deposit PEDOT directly into brain tissue from electrodes implanted into mouse brain slices (1-4 mm thick). The tissue slices were physically stabilized during electrode insertions and electropolymerization procedures by immobilization within gelatin $(10 \%$ in phosphate 


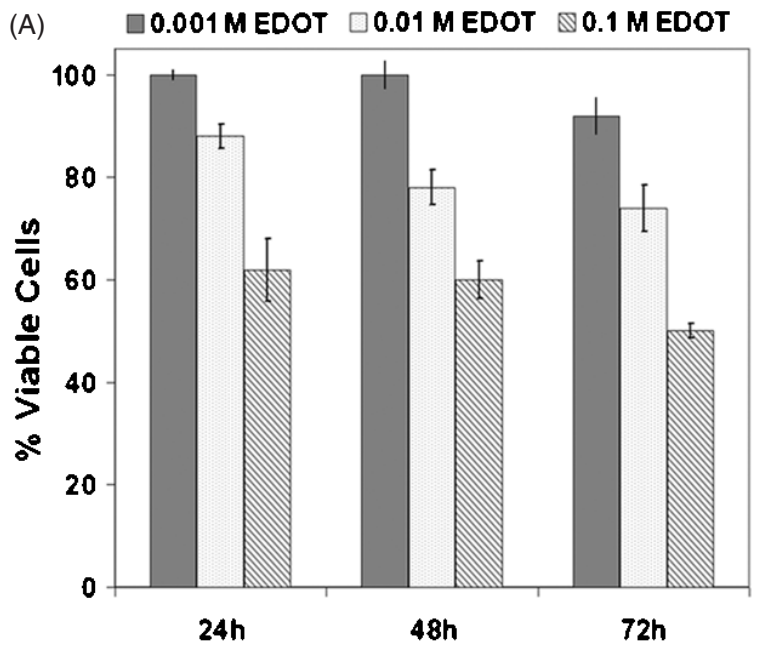

Hours After Exposure to EDOT monomer
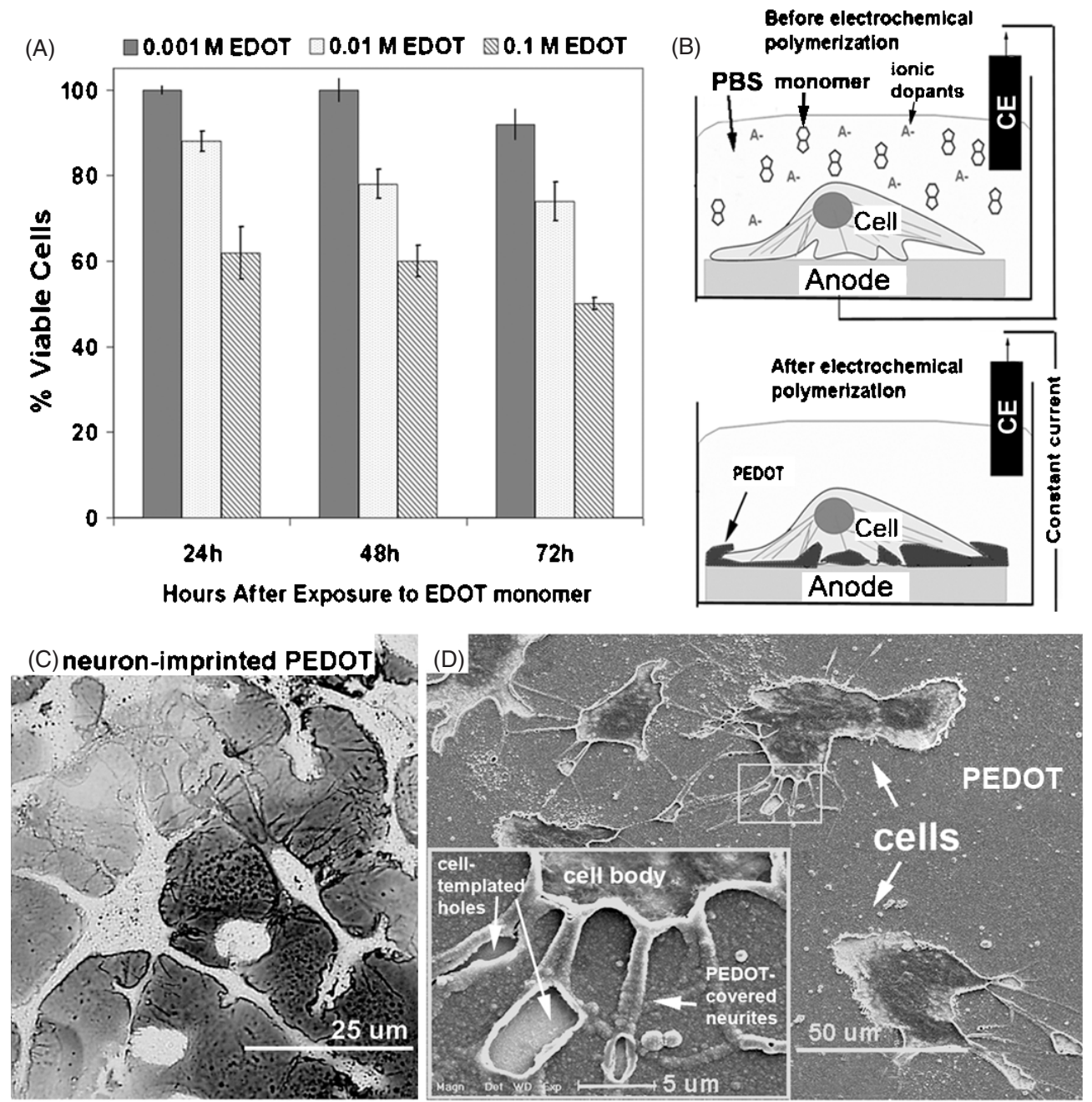

Figure 1. PEDOT can be polymerized in the presence of living neural cells resulting in a cell-templated, biomimetic conducting polymer matrix. (A) Time course of cell viability following exposure of SY5Y neural cells to increasing concentrations of EDOT monomer solution (0.001-0.1 M EDOT with 0.02 M PSS in PBS). Cell viability was determined by MTT assay using three independent cell samples for each time point and each concentration. (B) Diagram representing the process of electrochemical polymerization in the presence of living cells cultured on an electrode substrate. (C) Optical micrograph of PEDOT (dark substance) polymerized around live cells of mouse dissociated cortical neuronal cultures. The PEDOT intimately contacts the cell membranes revealing neurites, filopodia and nanoscale cellular processes. (D) Scanning electron micrograph (SEM) of PEDOT polymerized around neural cells reveals cell-shaped holes in the PEDOT matrix as well as cell-templated caves, tunnels and troughs. The nano and microscale fuzziness and roughness that is characteristic of PEDOT topology is also revealed by these SEM images. All cells were formaldehyde fixed prior to imaging and for SEM cells were also dehydrated with hexmethyldisilazane (HMDS).

buffered saline, PBS) (figure 2(a)). In order to stimulate PEDOT formation via electrochemical polymerization, EDOT monomer must be near the active electrode. Therefore, 0.01 M EDOT monomer (in PBS that also contained 0.02 M PSS) was delivered to the electrode sites within the brain slice in one of two ways; monomer solution was allowed to passively diffuse into the entire slice by submersion (10-20 $\mathrm{min}$ at room temperature) prior to electrode insertion or the monomer solution was injected by 29 gauge needle directly into the vicinity of the electrode tip after insertion. The working/stimulating electrodes (WE/SE) used for this study were single Teflon-coated gold microwires (75 $\mu \mathrm{m}$ diameter) with $1 \mathrm{~mm}$ of exposed, bare wire at the tip. A platinum wire reference/counter electrode (RE/CE) was then placed into the gelatin matrix or brain tissue, $10-20 \mathrm{~mm}$ away from the tip of the WE/SE (see figure 2(a)). Galvanostatic current $\left(0.5-5 \mu \mathrm{A} \mathrm{mm}^{-2}\right)$ was delivered using an Autolab Potentiosta/Galvanostat (EcoChemie, The Netherlands). The polymer first deposited on the electrode tip itself and then grew out into the tissue forming a diffuse, blue-gray spherical cloud of PEDOT within the brain parenchyma. To examine how the morphology and kinetics of formation of the PEDOT cloud varied with increasing amounts of charge, three different wires were inserted in the same tissue slice and deposition charges of $45 \mathrm{mC}, 90 \mathrm{mC}$ and $135 \mathrm{mC}$ were applied $(50 \mu \mathrm{A}$ for 15 , 30 or $45 \mathrm{~min}$, respectively) (figure 2(b)). First, the lateral extent and then the opacity of the PEDOT cloud increased systematically with the total charge applied. The pixel density (measured with ImageJ) of the PEDOT clouds resulting from $45 \mathrm{mC}, 90 \mathrm{mC}$ and $135 \mathrm{mC}$ were found to be $60 \%, 76 \%$ and 

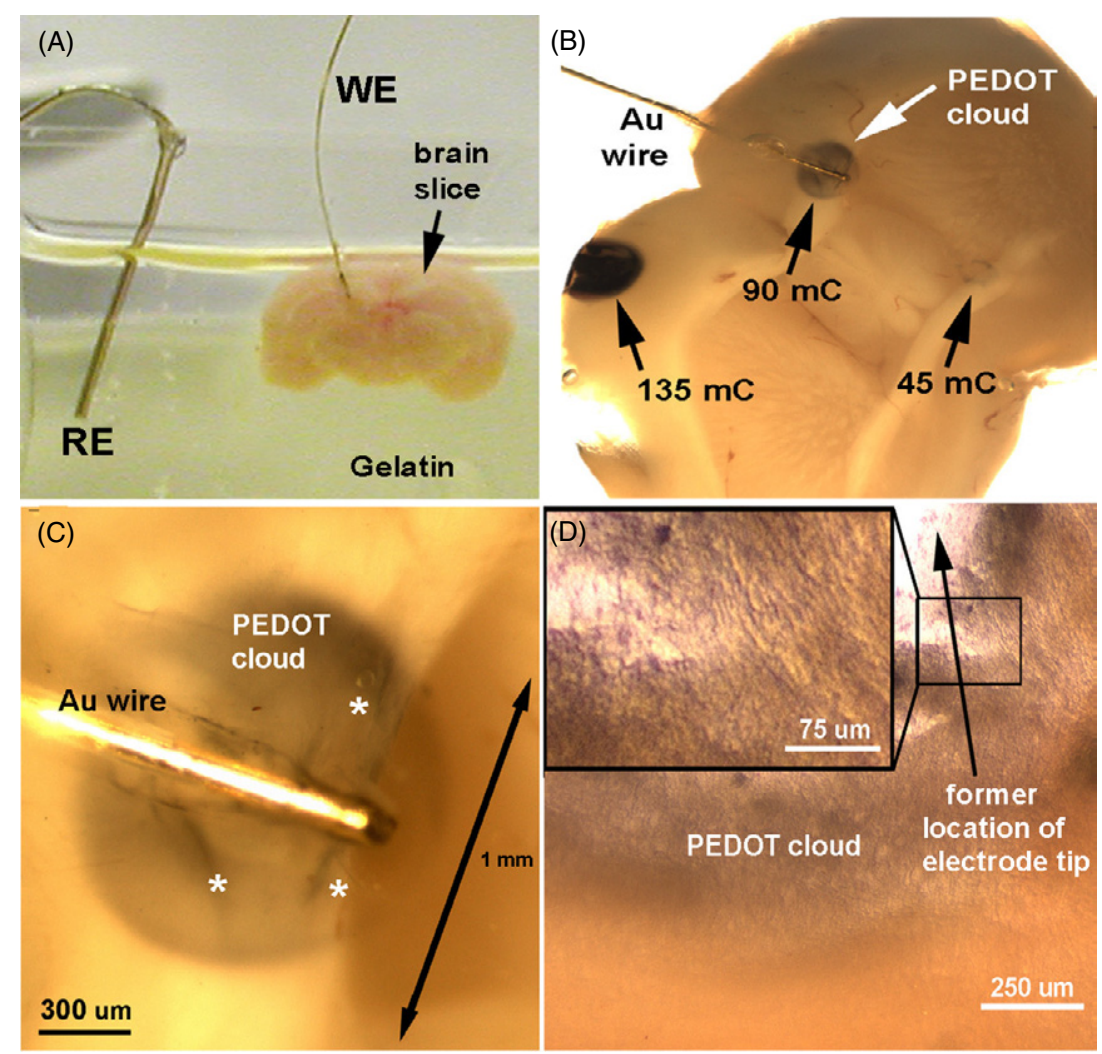

Figure 2. A network of conducting PEDOT filaments can be polymerized directly within brain tissue from an implanted electrode. (A) An image of the set-up used to polymerize PEDOT directly within a mouse brain slice. The tissue slice is physically stabilized within gelatin then a microwire electrode is inserted into the tissue and a platinum wire reference/counter electrode is inserted into the gelatin. (B) A mouse brain slice ( $2 \mathrm{~mm}$ thick) within which three distinct PEDOT networks were polymerized from a Au microwire using increasing polymer deposition charges of $45 \mathrm{mC}, 90 \mathrm{mC}$ and $135 \mathrm{mC}$. (C) A higher magnification image reveals that the PEDOT cloud integrates directly within the tissue and appears to intensify near and follow white matter fiber tracts (see white stars). (D) High magnification optical micrograph reveals the microscale structure of the PEDOT filaments within the network and suggests that tissue and extracellular matrix structures were used as a scaffold for polymerization. These data are representative of similar findings in roughly 20 brain slices in which PEDOT was polymerized using similar deposition conditions.

91\% saturated, respectively. PEDOT clouds could be grown throughout the brain and we found that the morphology of the PEDOT fibrils within the cloud appeared to be at least in part determined by the morphology of the tissue within the region of the brain in which the PEDOT was polymerized. This is evident in a higher magnification view of the PEDOT cloud resulting from the $90 \mathrm{mC}$ polymerization which reveals localized variations in optical density. These variations are presumably associated with PEDOT polymerization around specific structures within the brain tissue such as white matter tracts which acted as fibrous scaffolds for polymerization (see * (white stars) in figure 2(c)). Furthermore, the $90 \mathrm{mC}$ PEDOT cloud extended roughly $1 \mathrm{~mm}$ out into the tissue, exceeding the $100-150 \mu \mathrm{m}$ width of a glial scar that has been found to form around implanted neural electrodes (Polikov et al 2005) (see figure 2(c)). Higher magnification images near the electrode-tissue surface indicated that the PEDOT clouds were actually low-density networks of diffuse PEDOT that appeared to have grown within the extracellular and interstitial spaces and polymerized on extracellular matrix (ECM) components (figure 2(d)). This formed a net of PEDOT fibrils, chains and filaments woven around and between cells, effectively innervating the tissue.
PEDOT is not expected to be biodegradable and thus these conductive polymer networks are permanent, threedimensional electrode structures that fill some of the interstitial spaces and coat ECM components. PEDOT coatings and networks in gels have been found to be porous at the nano and microscale; however, it will clearly be necessary to establish that the presence of the PEDOT networks does not interfere with diffusion and local mass transport within the brain tissue that is needed for normal signal transduction and maintenance of cell viability (Kim et al 2004, Yang et al 2005). We are currently conducting experiments that assess how the polymerization procedure and the presence of the filamentous PEDOT network affects the neurons it contacts and those in the surrounding tissue. Preliminary studies in which we labeled cells within the PEDOT clouds with Nissl stain indicate that cells remain intact following polymerization and that local diffusion and mass transport were not disrupted by the presence of the PEDOT. Nissl labels a type of ribosomal RNA which is abundant within the cytoplasm of healthy neurons and some glial cells. We found that fluorescent Nissl stain (Molecular Probes) was taken up by cells within the PEDOT cloud as long as $3 \mathrm{~h}$ following PEDOT polymerization and following a series of electrical analyses on the tissue (figures 3(b) and (c)). 

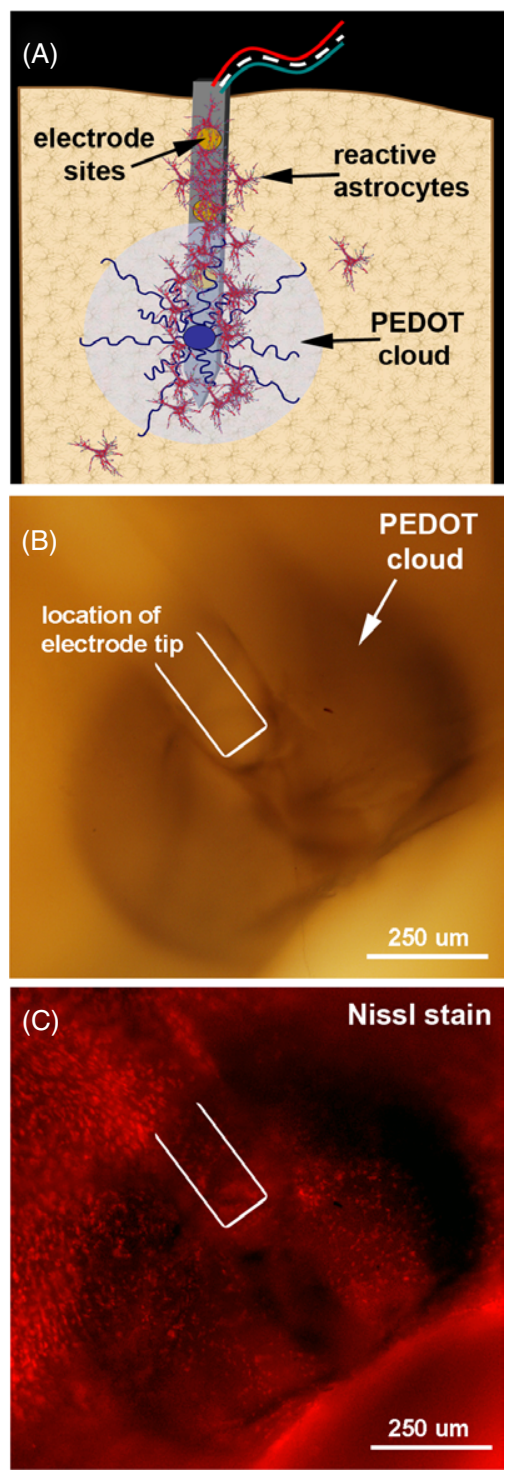

Figure 3. Cells within the PEDOT network remain intact and the PEDOT network is not a barrier to diffusion. (A) Diagram representing the process of polymerizing PEDOT directly into brain tissue from a neural electrode device in order to bypass the surrounding glial scar. (B) An optical micrograph of a PEDOT cloud deposited directly within brain tissue shows that the morphology of the brain tissue in the region in which PEDOT is polymerized affects the morphology of the PEDOT fibrils within the conductive cloud (see arrows). (C) The same tissue section was then exposed to fluorescent (red) Nissl stain indicating that the neurons within the PEDOT cloud are intact following polymerization. These data are representative of findings in three brain slices which underwent similar polymerization and staining procedures.

In order to serve as an electrode for an implanted device, it is necessary that this PEDOT network is electrically functional and be able to maintain long-term electrical connectivity while in a physically and biochemically dynamic environment. It should also be a highly sensitive electrode that can deliver amounts of charge relevant for communication with its target cells. To assess these issues, we performed both electrical impedance spectroscopy (EIS) to determine electrode impedance and cyclic voltammetry $(\mathrm{CV})$ to determine charge transfer capacity and identify any redox reactions that may occur during charge delivery procedures. A solution of 0.1 M PBS ( $\mathrm{pH} 7.0$ ) was used as the electrolyte in a three-electrode cell for both EIS and CV. A platinum foil was used as the counter electrode and a saturated calomel $\mathrm{Ag} / \mathrm{AgCl}_{2}$ electrode was used as the reference electrode. The PEDOT network electrode was connected to (and became) the working electrode. The values of the impedance were determined across frequencies ranging from 1 to $100000 \mathrm{~Hz}$ and the real and imaginary components of the impedance were measured as a function of frequency. For CV, a scan rate of $10 \mathrm{mV} \mathrm{s}^{-1}$ was used and the potential on the working electrode was swept between -1.0 and $1.0 \mathrm{~V}$ versus SCE. This limit was wide enough to include reversible redox reactions yet narrow enough to avoid over-oxidation and remain within the water window.

Electrodes were tested before, during and after polymerization. Similar to results from studies on PEDOT deposition within low-density hydrogels (Kim et al 2004), the filamentous PEDOT network deposited within brain tissue manifests in a large increase in effective surface area of the electrode as compared to that of the bare electrode as evidenced by decreased impedance and increased charge capacity. We found that the decrease in impedance, change in phase angle and increase in charge capacity of the PEDOT network scaled according to the total current applied during polymerization, specifically deposition charges of $45 \mathrm{mC}, 90 \mathrm{mC}$ and $135 \mathrm{mC}$ (see figures 4(a) and (c)). We performed a variety of control procedures, including application of electrical current in the absence of monomer solution (in PBS only) and this had little effect on the impedance or charge capacity of the electrode (see red lines in figures 4(a)-(c)).

For all three deposition charges, the presence of the PEDOT network decreased the electrical impedance by $1-2$ orders of magnitude and reduced the phase angle of the impedance from $60^{\circ}$ for bare wire to about $10^{\circ}$ (figures 4 (a) and (b)). Previous studies on PEDOT-coated neural electrodes indicate that similar changes in impedance are associated with increased electrode conductivity and sensitivity that result in an increase in signal-to-noise ratio and number of detectable spikes during in vivo neural recordings (Cui et al 2003, Ludwig et al 2006). Furthermore, biomedical device electrodes with low impedance generally have lower overall power requirements (Lanmuller et al 1997, Markewitz et al 1995). For implanted devices this manifests in extended battery life and provides an opportunity for use of alternative device power strategies such as telemetry and bio-powering (Ebron et al 2006, Gorge et al 2001, Neihart and Harrison 2005).

The PEDOT networks within the brain tissue are also associated with an increase in charge transfer capacity of the electrode and a dramatic change in shape and symmetry of the CV curve (figure 4(c)). The increase in area under the $\mathrm{CV}$ curve for the PEDOT networks as compared to the bare electrode indicates increased charge transfer capabilities and is likely associated with the increased effective electrode surface area (Heien et al 2003, Merrill et al 2005). The asymmetric shape of the voltammograms for the PEDOT networks formed 


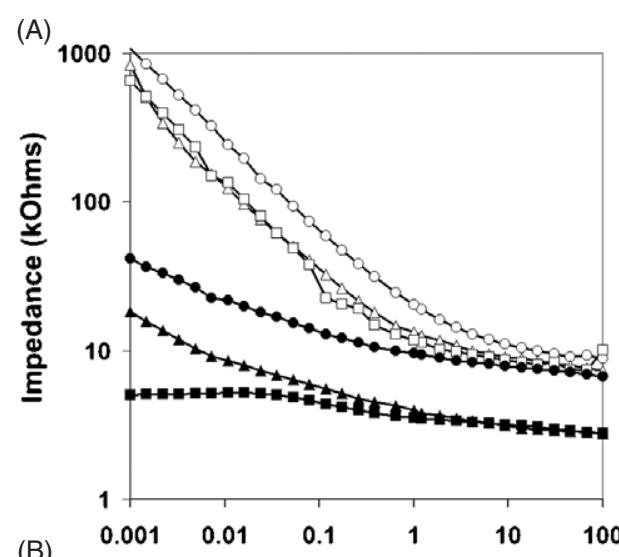

(B)
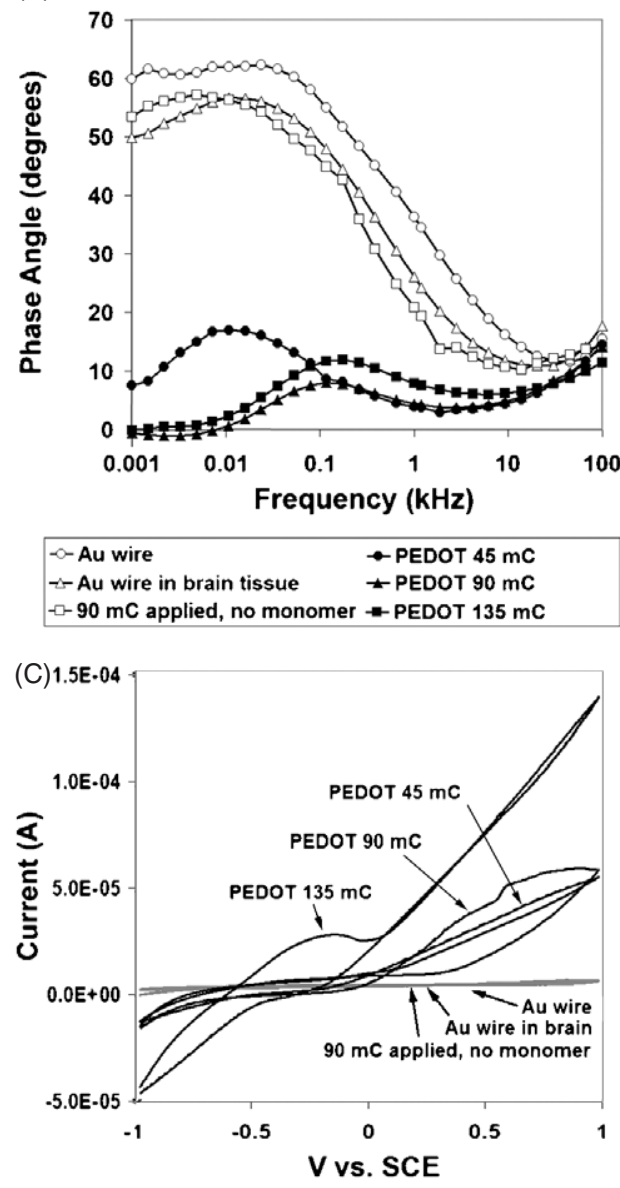

Figure 4. The filamentous PEDT network polymerized directly within brain tissue is a functional electrode with lower impedance and increased charge capacity compared to the bare electrode.

(A) EIS comparing the impedance spectra for the Au microwire before (open circles) and after insertion into brain tissue (open triangles) to that of three distinct PEDOT networks polymerized from the wire into the tissue (increasing deposition charge from filled circles, filled triangles, to filled squares). For the control electrical current was applied to the brain tissue via the wire in the absence of monomer (open squares). (B) The phase angle of the impedance as detected by EIS. (C) CV curves comparing the charge capacity for the Au microwire before and after insertion into brain tissue to that of three distinct PEDOT networks polymerized from the wire into the tissue and the control. For all electrical analyses, a platinum wire and foil was used as the counter electrode and a saturated calomel $\mathrm{Ag} / \mathrm{AgCl}$ is the reference electrode. These data are representative of similar findings in roughly ten brain slices in which PEDOT was polymerized using similar deposition conditions. within the brain tissue suggest that irreversible Faradaic reactions are occurring as the current direction is reversed. This is likely associated with diffusion of electrochemical species away from the electrode network (Merrill et al 2005) but could also be an indication that the presence of the PEDOT network reduces ion mobility within the tissue (Yildiz et al 1999). For reversible Faradaic reactions the direction of electrochemical reactions are reversed to minimize unrecoverable charge by maintaining a zero net accumulation of electrochemical species. In contrast irreversible reactions are usually associated with reduced charge transfer capacity due to physical and electrical degradation of the electrode over time as well as possible tissue damage due to hydrolysis and oxidation reactions (Halliwell 1992, Merrill et al 2005, Stohs and Bagchi 1995). Fortunately, a diversity of alternative electrode stimulation and charge transfer methods that reduce unrecoverable charge and avoid production of unwanted electrochemical species are available for use in future studies of the PEDOT networks. One such method is cycling the electrode with a charge imbalanced biphasic waveform which takes into account the probability of irreversible redox reactions to minimize damage to both the tissue and the electrode (Merrill et al 2005).

In order to perform in vivo testing and analysis in defined animal models, a process for polymerizing these polymer networks within the context of neural electrode devices must be established. Local monomer delivery to the electrode sites could be accomplished in a variety of ways such as by local intracranial injections, infusions through hollow fibers (Broadhead et al 2002) or microcannulae placed in the tissue or via devices enabled with microfluidic channels (Rathnasingham et al 2004). In addition, it will be important that the fluid delivery method allows for optimization of the conditions needed to maintain monomer delivery at a rate consistent with its electrochemical deposition.

\section{Conclusions}

Polymerization of PEDOT directly within brain tissue results in formation of a conductive network that innervates the tissue and enables uniquely intimate, sensitive contacts between the electrode and the plasma membranes of neural cells. The high surface area of these PEDOT networks should facilitate signal transduction between the ionically conductive tissue and the electronically conductive device and transport electronic charge farther out into the tissue, hopefully eliminating the need to have the neurons near the electrode-tissue interface where immunoreactions are strongest and the mechanical stresses and strains are the highest. In addition to these studies in brain tissue, we also found that functional PEDOT networks could be polymerized within a variety of other tissues including dermis and cardiac muscle (data not shown). Due to the ability to grow within and through nano and micrometer thin spaces between cells for diameters of as much as $500 \mu \mathrm{m}$ to $1 \mathrm{~mm}$ from the electrode site, this type of conducting polymer electrode network may be able to penetrate fibrous scar encapsulations and bypass congregations of immune cells 
that often form around implanted electrodes throughout the body.

This technology may make possible the establishment of functional, long-term electrical communication between implanted biomedical devices and the healthy target cells tissue despite encapsulation of the device in high electrical impedance, signal blocking scar tissue. It can be adapted to create fully-integrated and more efficacious implanted electrodes for diverse applications such as neural recording/stimulation, peripheral nerve electrodes, cardiac anti-arrhythmia devices, muscle stimulation, voltage-mediated tissue ablation, $\mathrm{pH}$ monitoring, glucose sensing, cochlear implants and retinal prosthetics. In addition, the diffuse, electrically connected conductive polymer minimizes the necessity of stiff, sharp immunoreactant metal or alloy-based devices and signifies a new electrode paradigm built around an adaptive, filamentous conducting polymer network that innervates the living tissue.

\section{Acknowledgments}

The authors would like to thank Dong-Hwan 'Richie' Kim and Laura Povlich, who contributed to initial studies related to PEDOT polymerization in the presence of living cells, Dotty Sorenson and Shelly Almburg at the U of M Microscopy and Image Analysis Laboratory (MIL), and Dr John Mansfield and Dr Kai Sun at the U of M Electron Microscopy Analysis Laboratory (EMAL). Aspects of this research have been filed as a Provisional Patent with the USPTO and there are currently discussions underway to commercialize or license aspects of this technology. Dr Richardson-Burns has been supported by $\mathrm{NIH}$ institutional postdoctoral training grant T90-DK070071-02 and is now supported by NIH individual postdoctoral training grant F32 NS054618-01. This work has been supported in part by the NSF DMR-0084304, NSF DMR0518079 and NIH NINDS NO1-NS-1-2338 and will also be supported by Army MURI 50376.

\section{References}

Biran R, Martin D C and Tresco P A 2005 Neuronal cell loss accompanies the brain tissue response to chronically implanted silicon microelectrode arrays Exp. Neurol. 195 115-26

Brahim S and Guiseppi-Elie A 2005 Electroconductive hydrogels: electrical properties of polypyrrole-poly(HEMA) and electrochemical composites Electroanalysis 17 556-70

Broadhead K W, Biran R and Tresco P A 2002 Hollow fiber membrane diffusive permeability regulates encapsulated cell line biomass, proliferation, and small molecule release Biomaterials 23 4689-99

Cui X, Wiler J, Dzaman M, Altschuler R A and Martin D C 2003 In vivo studies of polypyrrole/peptide coated neural probes Biomaterials 24 777-87

Ebron V H et al 2006 Fuel-powered artificial muscles Science 311 1580-3

Edell D J, Toi V V, McNeil V M and Clark L D 1992 Factors influencing the biocompatibility of insertable silicon microshafts in cerebral cortex IEEE Trans. Biomed. Eng. 39 635-43

Eisen M D and Franck K H 2005 Electrode interaction in pediatric cochlear implant subjects J. Assoc. Res. Otolaryngol. 6 160-70
Fromherz P 2002 Electrical interfacing of nerve cells and semiconductor chips ChemPhysChem 3 276-84

Gilletti A and Muthuswamy J 2006 Brain micromotion around implants in the rodent somatosensory cortex J. Neural Eng. 3 189-95

Gorge G, Kirstein M and Erbel R 2001 Microgenerators for energy autarkic pacemakers and defibrillators: fact or fiction? Herz $\mathbf{2 6}$ 64-68

Halliwell B 1992 Reactive oxygen species and the central nervous system J. Neurochem. 59 1609-23

Heien M L, Phillips P E, Stuber G D, Seipel A T and Wightman R M 2003 Overoxidation of carbon-fiber microelectrodes enhances dopamine adsorption and increases sensitivity Analyst 128 1413-9

Jain A, Kim Y T, McKeon R J and Bellamkonda R V 2006 In situ gelling hydrogels for conformal repair of spinal cord defects, and local delivery of BDNF after spinal cord injury Biomaterials 27 497-504

Johnson M D, Otto K J and Kipke D R 2005 Repeated voltage biasing improves unit recordings by reducing resistive tissue impedances IEEE Trans. Neural Syst. Rehabil. Eng. $13160-5$

Khor E, Li H C and Wee A 1995 In situ polymerization of pyrrole in animal tissue in the formation of hybrid biomaterials Biomaterials 16 657-61

Kim D H, Abidian M and Martin D C 2004 Conducting polymers grown in hydrogel scaffolds coated on neural prosthetic devices J. Biomed. Mater. Res. A 71A 577-85

Kim D H and Martin D C 2006 Sustained release of dexamethasone from hydrophilic matrices using PLGA nanoparticles for neural drug delivery Biomaterials 27 3031-7

Kim B C, Spinks G M, Wallace G G and John R 2000 Electroformation of conducting polymers in a hydrogel support matrix Polymer 41 1783-90

Lanmuller H, Bijak M, Mayr W, Rafolt D, Sauermann S and Thoma H 1997 Useful applications and limits of battery powered implants in functional electrical stimulations Artif. Organs $21210-12$

Lee H, Bellamkonda R V, Sun W and Levenston M E 2005 Biomechanical analysis of silicon microelectrode-induced strain in the brain J. Neural Eng. 2 81-9

Ludwig K A, Uram J D, Yang J, Martin D C and Kipke D R 2006 Chronic neural recordings using silicon microelectrode arrays electrochemically deposited with a poly $(3,4-$ ethylenedioxythiophene) (PEDOT) film J. Neural Eng. 3 59-70

Markewitz A, Kronski D, Kammeyer A, Kaulbach H, Weinhold C, Doering W and Reichart B 1995 Determinants of dual chamber pulse generators longevity Pacing Clin. Electrophysiol. 18 2116-20

Merrill D R, Bikson M and Jefferys J G 2005 Electrical stimulation of excitable tissue: design of efficacious and safe protocols $J$. Neurosci. Methods 141 171-98

Neihart N M and Harrison R R 2005 Micropower circuits for bidirectional wireless telemetry in neural recording applications IEEE Trans. Biomed. Eng. 52 1950-9

Nyberg T, Inganas O and Jerregard H 2002 Polymer hydrogel microelectrodes for neural communication Biomed. Microdevices 4 43-52

Otto K J, Johnson M D and Kipke D R 2006 Voltage pulses change neural interface properties and improve unit recordings with chronically implanted microelectrodes IEEE Trans. Biomed. Eng. $53333-40$

Patil P G, Nicolelis M A L and Turner D A 2004 Brain-machine interface: ensembles of human subcortical neurons and signals for neuroprosthetic control J. Neurosurg. 100796

Polikov V S, Tresco P A and Reichert W A 2005 Response of brain tissue to chronically implanted neural electrodes J. Neurosci. Methods 148 1-18 
Rathnasingham R, Kipke D R, Bledsoe S C and McLaren J D 2004 Characterization of implantable microfabricated fluid delivery devices IEEE Trans. Biomed. Eng. 51 138-45

Richardson-Burns S M, Hendricks J L, Foster B, Povlich L K, Kim D H and Martin D C 2007 Polymerization of the conducting polymer poly(3,4-ethylenedioxythiophene) (PEDOT) around living neural cells Biomaterials 28 1539-52

Rodriguez-Oroz M C, Zamarbide I, Guridi J, Palmero M R and Obeso J A 2004 Efficacy of deep brain stimulation of the subthalamic nucleus in Parkinson's disease 4 years after surgery: double blind and open label evaluation J. Neurol. Neurosurg. Psychiatry 75 1382-5

Rousche P J and Normann R A 1998 Chronic recording capability of the Utah Intracortical Electrode Array in cat sensory cortex J. Neurosci. Methods 82 1-15

Rousche P J, Pellinen D S, Pivin D P, Williams J C, Vetter R J and Kipke D R 2001 Flexible polyimide-based intracortical electrode arrays with bioactive capability IEEE Trans. Biomed. Eng. 48 361-71

Spataro L, Dilgen J, Retterer S, Spence A J, Isaacson M, Turner J N and Shain W 2005 Dexamethasone treatment reduces astroglia responses to inserted neuroprosthetic devices in rat neocortex Exp. Neurol. 194 289-300

Stieglitz T, Schuettler M and Koch K P 2005 Implantable biomedical microsystems for neural prostheses IEEE Eng. Med. Biol. Mag. 24 58-65

Stohs S J and Bagchi D 1995 Oxidative mechanisms in the toxicity of metal ions Free Radic. Biol. Med. 18 321-36

Subbaroyan J, Martin D C and Kipke D R 2005 A finite-element model of the mechanical effects of implantable microelectrodes in the cerebral cortex J. Neural Eng. 2 103-13

Szarowski D H, Andersen M D, Retterer S, Spence A J, Isaacson M, Craighead H G, Turner J N and Shain W 2003 Brain responses to micro-machined silicon devices Brain Res. 983 23-35
Turner J N, Shain W, Szarowski D H, Andersen M, Martins S, Isaacson M and Craighead H 1999 Cerebral astrocyte response to micromachined silicon implants Exp. Neurol. $15633-49$

Vetter R J, Williams J C, Hetke J F, Nunamaker E A and Kipke D R 2004 Chronic neural recording using silicon-substrate microelectrode arrays implanted in cerebral cortex IEEE Trans. Biomed. Eng. 51 896-904

Williams J C, Holecko M M, Massia S P, Rousche P and Kipke D R 2005 Multi-site incorporation of bioactive matrices into MEMS-based neural probes J. Neural Eng. 2 L23-8

Williams J C, Rennaker R L and Kipke D R 1999 Long-term neural recording characteristics of wire microelectrode arrays implanted in cerebral cortex Brain Res. Protoc. 4 303-313

Xiao Y H, Cui X Y, Hancock J M, Bouguettaya M B, Reynolds J R and Martin D C 2004 Electrochemical polymerization of poly(hydroxymethylated-3,4-ethylenedioxythiophene) (PEDOT-MeOH) on multichannel neural probes Sensors Actuators B 99 437-43

Yang J Y, Kim D H, Hendricks J L, Leach M, Northey R and Martin D C 2005 Ordered surfactant-templated poly(3,4-ethylenedioxythiophene) (PEDOT) conducting polymer on microfabricated neural probes Acta Biomaterialia 1 125-36

Yang J Y and Martin D C 2006 Impedance spectroscopy and nanoindentation of conducting poly(3,4-ethylenedioxythiophene) coatings on microfabricated neural prosthetic devices J. Mater. Res. 21 1124-32

Yildiz G, Catalgil-Giz H and Kadirgan F 1999 Electrochemically prepared acrylamide $/ N, N^{\prime}$-methylene bisacrylamide gels J. Appl. Electrochem. 30 71-5

Zhong Y H and Bellamkonda R V 2005 Controlled release of anti-inflammatory agent alpha-MSH from neural implants J. Control. Release 106 309-18 